\title{
FRFT Low Pass Filter for Time delayed Signals
}

\author{
${ }^{1}$ P.V.Muralidhar and ${ }^{2}$ S.K.NAYAK
}

\begin{abstract}
In this paper we have been implement time delay signals response for Finite Impulse Response(FIR) Low Pass Filter using Fractional Fourier Transform (FRFT) based Kaiser window and also a methodology to tune the spectral characteristics of a linear phase FIR filer by changing the parameter of Fractional Fourier Transform. Here the signal to noise ratio is calculated for delayed version of input signals for proposed filter.
\end{abstract}

\section{INTRODUCTION}

The time-delay is fundamental issue in many signal processing areas including Radar, Sonar, communication and ultrasonic . The objective of Time-delay is to determine the delay of the corrupted received signals from the target. In literature different FIR windows like Hanning, Hamming, Rectangular are designed using FRFT[1-4]. In this paper we designed Time delay FRFT LPF Rectangular from FRFT Kaiser window and also a methodology to tune the transition band of a linear band of a linear phase. Finite impulse response (FIR) low pass Kaiser window using Fractional Fourier Transform (FRFT) is proposed. The merit of using FRFT in FIR low pass design is that the impulse response need not be recomputed to improve filter tuning and this online improvement in frequency response can be obtained. If a signal $s(t)$ delayed by $\tau$, the FRFT write angle ' $L$ ' of the delayed signal $\mathrm{s}(\mathrm{t}-\tau)$ can be expressed as [1] $\bar{S}_{\alpha}(u)=S_{\alpha}(u-\tau \cos \alpha) e^{\left[\hat{j} \tau^{2 \frac{\sin \alpha \operatorname{Cos} \alpha}{2}-j u \tau \sin \alpha}\right]}$

contrast to case the conventional FT, the delay property of the FRFT can be with respect to the location of extremism of the FRFT spectrum. Since FRFT has a fine concentration for chirp signals, the time-delay estimate for chirp signals becomes feasible by applying the delay property of the FRFT. In this paper, we present FRFT based LPF response for different delayed input signal and calculated SNR ratios of different delayed input signals.

\section{II. . FRACTIONAL FOURIER TRANSFORM}

\section{(FRFT):}

In mathematics, the Fractional Fourier Transform (FRFT) is a linear transformation generalizing the Fourier Transform. It can be through of as the Fourier Transform to the $n$-the power where $n$ need not be an integer. Thus it can transform a function to an intermediate domain between time and frequency.

1Assoc.prof. E.C.E. Dept. AITAM engg. College,TEKKALI

2 Berhampur university,BERHAMPUR.
A generalization of Fourier Transform, the Fractional Fourier Transform was first introduced by Victor Namias in 1980 [7]. The Fractional Fourier Transform X, of a function x, with an angle $\alpha$, [4]is defined by means of the Transformation kernel $K_{a}(t, u)$. $X_{a}(u)$ can be expressed as

$$
F^{a}[f(x)]=\int_{-\infty}^{\infty} K^{a}\left(x, x_{a}\right) f(x) d x=f^{a}\left(x_{a}\right)
$$

Where

$$
\begin{aligned}
& K^{a}\left(x, x_{a}\right)=\exp \left\lfloor i \pi\left(x^{2} \cot \alpha-2 x x_{a}+x_{a}^{2} \cot \alpha\right)\right\rfloor \\
& \text { and } \alpha=a \pi / 2
\end{aligned}
$$

Function $f(x)$ in a domain ' $\mathrm{x}$ ' to a function $f^{a}\left(x_{a}\right)$ in another domain ' $x_{a}$ '.

An FRFT with $\alpha=\pi / 2$ corresponds to the classical Fourier transform, and one with $\alpha=0$ corresponds to the identity operator. FRFT order can also be used in place of FRFT angle. The relationship between FRFT order and abgle is given by $\alpha=a \pi / 2$. In signal processing, FRFT has been applied to communications.

\section{DESIGN OF WINDOWS USING KAISER:}

Window functions has been successfully using in various areas of filtering, beam focusing signal processing and communications etc,. The role of windows is quite impressive and economical from the parts of computational complexity and ease is associated with its application. The study of Side lobe fall of ratio (SLFOR), main side lobe level (MSLL), half main lobe width (HMLW) for different windows functions for different values of ' $a$ ' $[5,6]$. Window function is related based on the parameter $\beta$ for a Kaiser window that emulates the corresponding window properties. The Kaiser window based on the first order Bessel's function is special in two respects. It is nearly optimal in terms of the relationship between "RINGING" suppression and transition width and second, it can be tuned by $\beta$ which determines the ringing of the filter. $\beta$ is the window shape parameter.

\section{IMPLEMENTATION OF LOW PASS FILTER USING CONVOLUTION:}

In this method we proposed to tune procedure based on transition characteristics of filters using Convolution method .The technique used for tuning the transition bandwidth of low pass FIR filters is by using FRFT.

Tuning procedure of FIR Lowpass filters using Frft:

An FIR digital filter operation is a linear convolution of the finite duration impulse response with the input signal sequence $x(n)$. The filtering operation in the frequency domain 
is done using the FFT/IFFT algorithms . Impulse response, $h$ $(n)$, for Kaiser window.

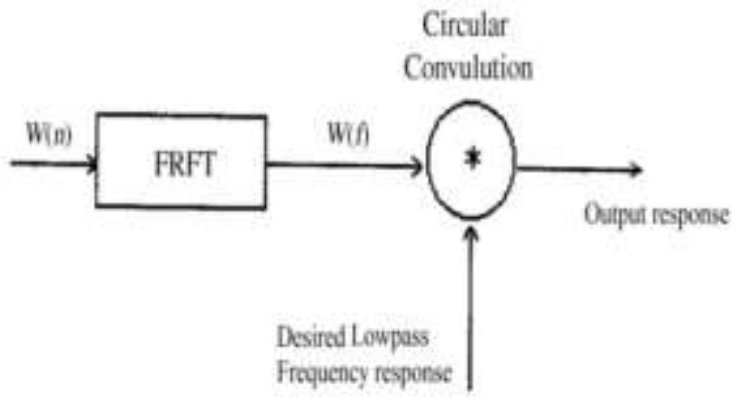

Fig. 4.1: block diagram of tuning procedure

FIR filter is given by

$h(n)=h_{d}(n) w(n)$

Where $h_{d}(n)$ is the desired or ideal impulse response, and $w$ $(n)$, the Kaiser window sequence. Since multiplication in the time domain corresponds to convolution in the frequency domain, can be expressed as a complex convolution operation [8] given by

$$
H(\omega)=\frac{1}{2 \pi} \int_{-\pi}^{\pi} W(\lambda) H_{d}(\omega-\lambda) d \lambda,
$$

Where, $H(\omega)$ is the frequency response of filter, $H_{d}(\omega)$, the desired or ideal frequency response(of low pass filter), and $W(\omega)$, the frequency response of Kaiser window.

\section{Delay:10}

$\mathrm{a}=.1$ :

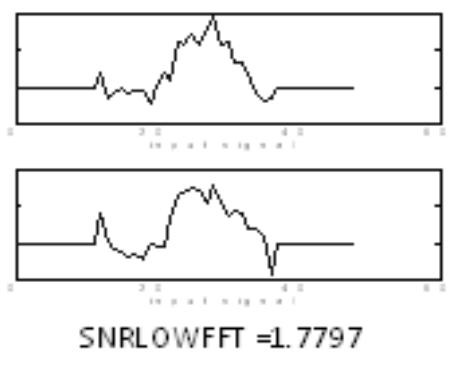

Fig 5.1.a

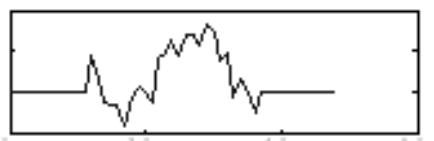

$\mathrm{a}=.5$ :

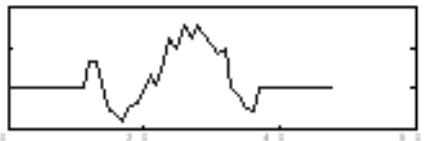

SNRLO WFFT $=2.0322$

Fig. 5.2. a
Thus, $H(\omega)$ is a circular or periodic convolution of the desired frequency response of low pass filter with the Fourier transform of the window function. As a result, the width of the transition band of $H(\omega)$ is proportional to the main lobe width of $W(\omega)$. From Fig it is observed that as the FRFT order is reduced the main lobe width of window's FRFT shrinks. This feature has been used here to tune the transition width and the filtering scheme is shown in fig. By varying the FRFT order filter, transition width can be changed.

For many applications, the input signal sequence is of long duration compared to the filter impulse response duration. The proposed tunable filtering scheme performs filtering in frequency domain. In this scheme, in place of impulse response coefficients, window function coefficients, $w(n)$, are computed corresponding to the initial filtering requirements. FFT of $w(n)$ is then obtained by taking FRFT order $a=1$, and is circularly convolved with the desired frequency response, to obtain $H$ (f), which is stored to perform filtering. If required, filter frequency response can be sharpened by changing the FRFT order, thus modifying the $H$ (f). Hence, in the proposed work filter response coefficients are not obtained in the time domain.

\section{Results}

Results for direct method of tuning using Kaiser window for different delayed input sequences responses are shown in fig. 5.1.a \& b to 5.12.a \& b. Here we have applied different delayed input like delay by $1,10 \& 20$ samples for different values of $\alpha=a \Pi / 2$ by selecting $\quad$ ' $a$ ' $=0.1,0.5,0.9 \& 1$. And also calculated different FRFT values signal to noise ratio are observed from the following figures.

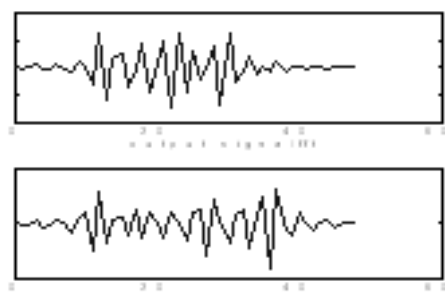

SNRLOWFRFT $=3.1314$

Fig. 5.1. b
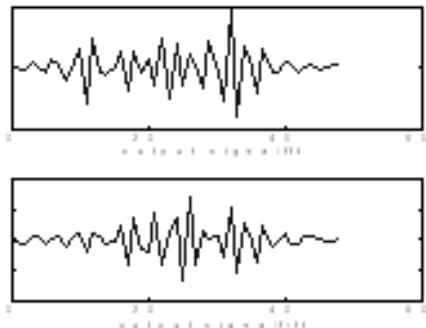

SNRLOWFRFT $=2.6981$

Fig. 5.2.b

$\mathrm{a}=.9$ 

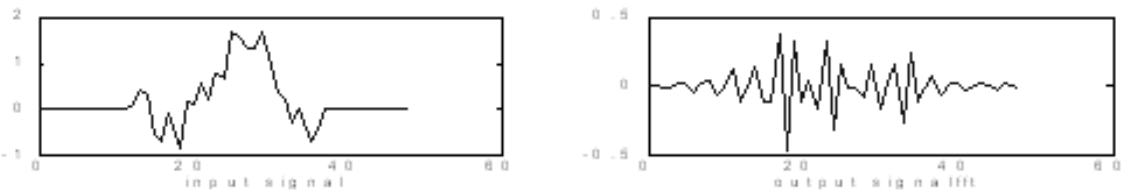

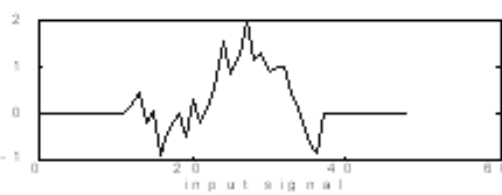

SNRLOWFFT $=2.4011$

Fig. 5.3. a
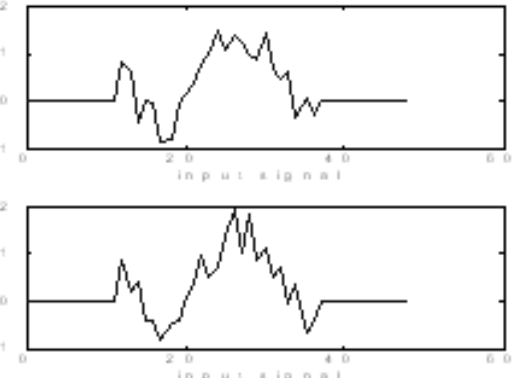

SNRLOWFFT $=2.3073$

Fig. 5.4. a

\section{Delay 20}

$\mathrm{a}=.1$
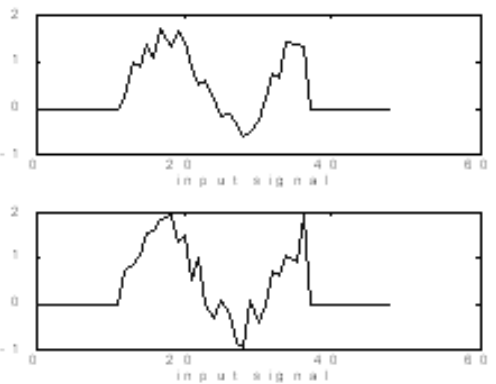

SNRLOWFFT $=1.8696$

Fig. 5.5. a
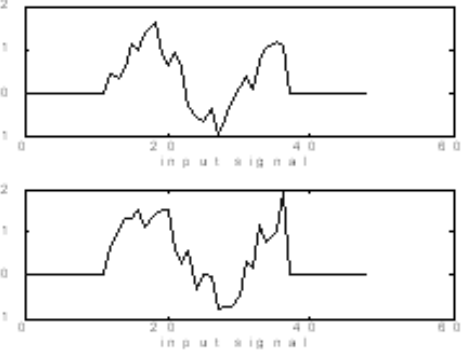

SNRLOWFFT $=2.6975$

Fig. 5.6. a

$\mathrm{a}=.5:$

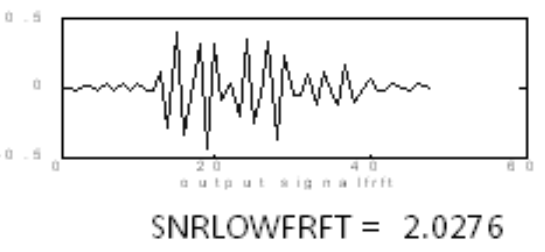

Fig. 5.3.b

$=1$
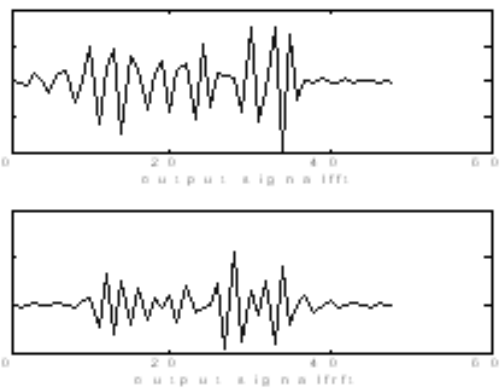

SNRLOWFRFT $=3.0086$

Fig. 5.4.b

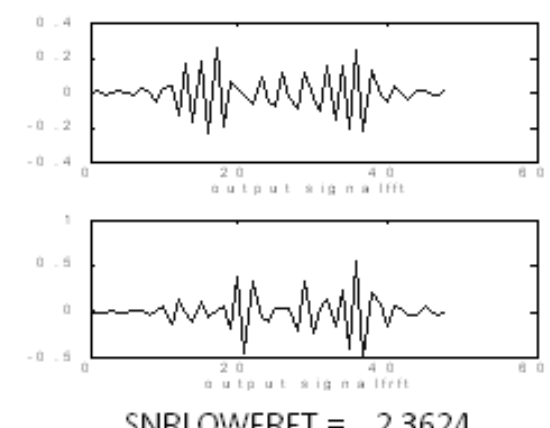

SNRLOWFRFT $=2.3624$

Fig. 5.5.b
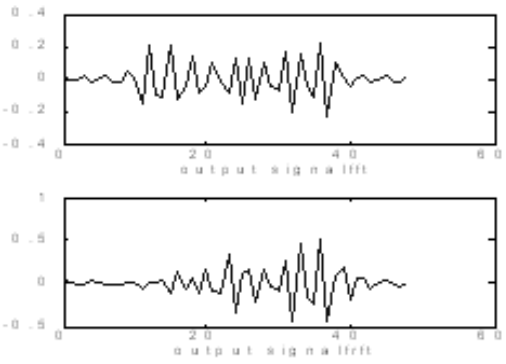

SNRLOWFRFT $=2.0572$

Fig. 5.6.b 

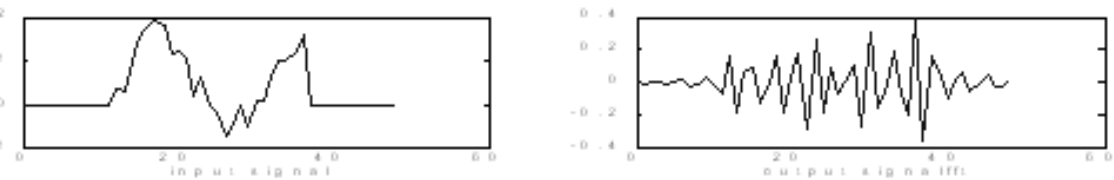

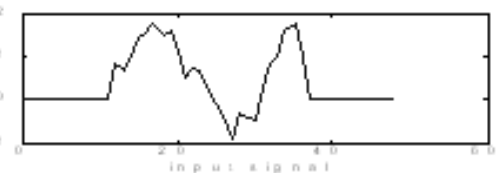

SNRLOWFFT $=2.2226$

Fig. 5.7. a
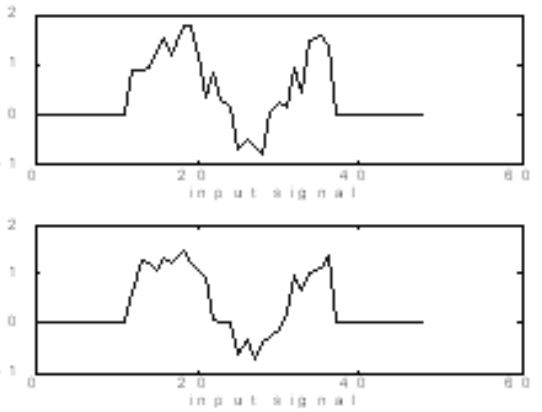

SNRLOWFFT $=2.0366$

Fig. 5.8. a

Delay 1
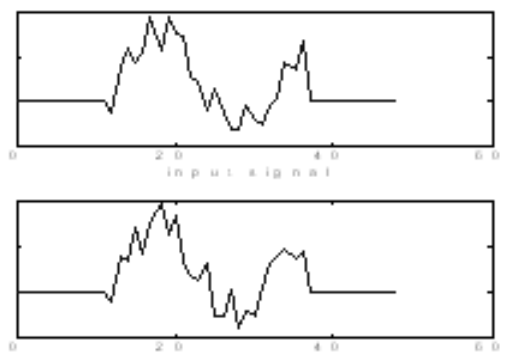

SNRLOWFFT $=2.3037$

Fig. 5.9. a
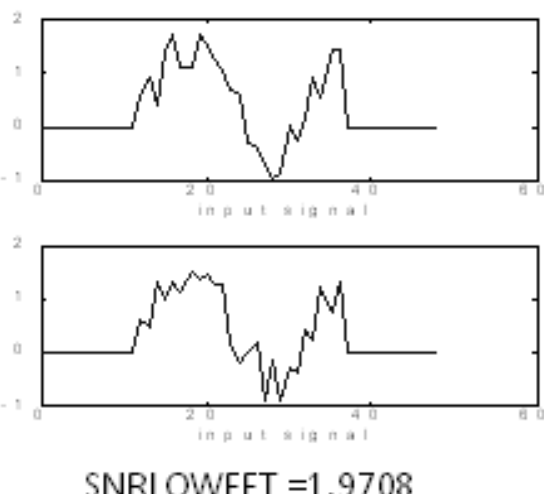

Fig. 5.10. a $\mathrm{a}=.1$ :

$\mathrm{a}=1:$

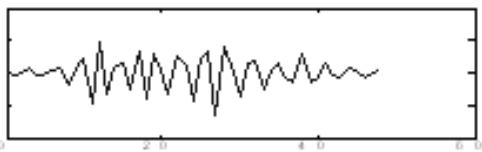

SNRLOWFRFT $=2.3262$

Fig. 5.7.b
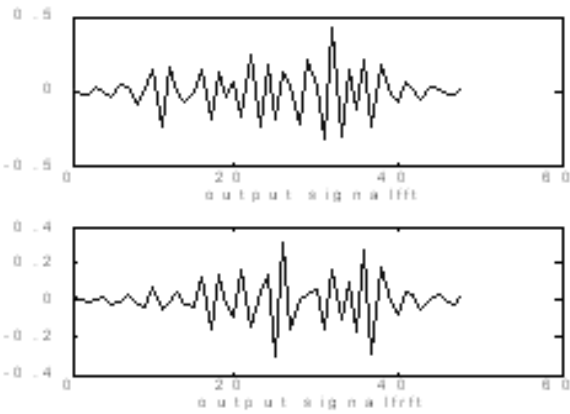

SNRLOWFRFT $=2.9342$

Fig. 5.8.b

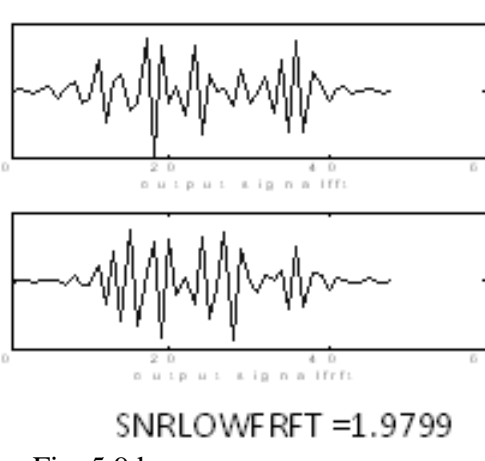

Fig. 5.9.b

$\mathrm{a}=.5$ :
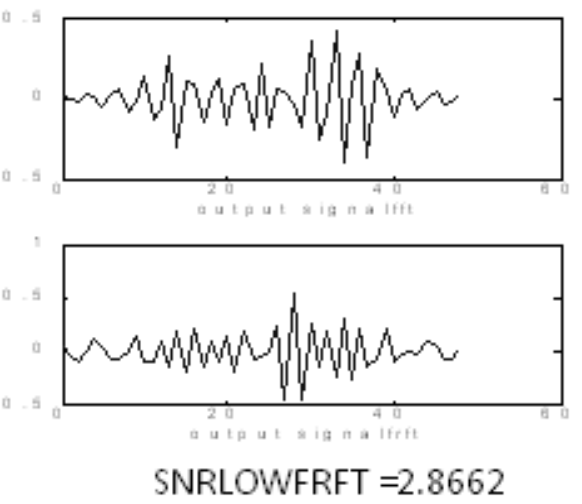

Fig. 5.10.b 


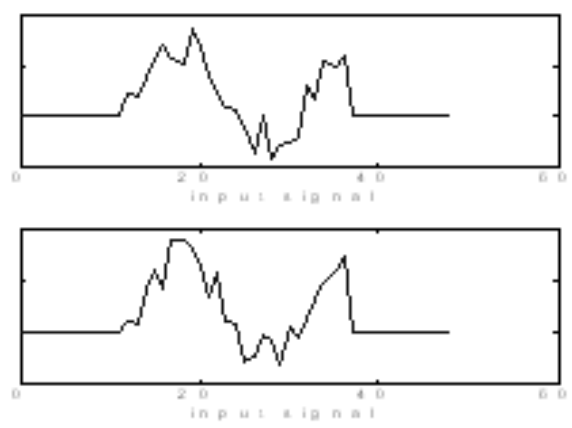

SNRLOWFFT $=2.5248$

Fig. 5.11. a

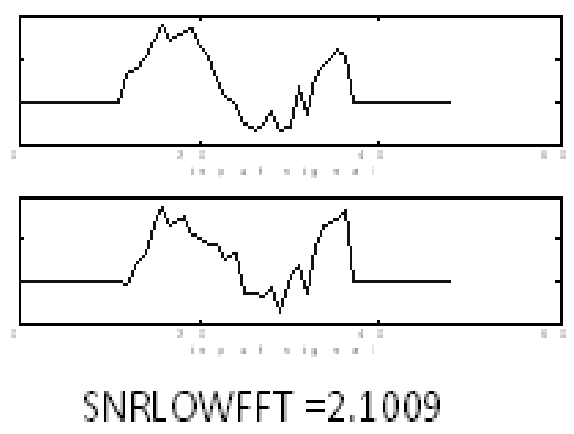

Fig. 5.12. a

\section{CONCLUSION}

From the figures we conclude that

1. For fixed time delay as the 'a' changing from 0.1 to 1 the signal to noise ratio changing.

2. For different time delays that is from $1,10 \& 20$ samples the signal to noise ratio is also changing. It is having medium SNR values for delay 1 and high values for medium delay values i.e., delay 10 and lower SNR values for delay 20

\section{REFERENCES}

[1] A.H.Quazi, "An overview on the time delay estimate in active and passive systems for target localization," IEEE Trans. Signal Process., vol.29, no. 6, pp. 527-533, Jun.1981.

https://doi.org/10.1109/TASSP.1981.1163618

[2] C.H.Knapp and G.C.Carter, " The generalized correlation method for estimation of time delay," IEEE Trans. Acoust., Speech, Signal Process., vol. 42, no.11, pp. 3084-3091, Nov. 1994.

[3] L.B.Almedia, " The fractional Fourier transform and time-frequency representations," IEEE Trans. Signal Process., vol.42, no.11, pp.30843091, Nov.1994.

https://doi.org/10.1109/78.330368

[4] H.M.Ozaktas, O.A rikan, M.A.Kutay, and G.Bozdagi, "Digital computation of the fractional Fourier transform," IEEE Trans. Signal Process., vol.44, no. 9, pp.2141-2150, sep.1996.

https://doi.org/10.1109/78.536672

[5] P.V.Muralidhar, A.S.SrinivasaRao, S.K.Nayak," Spectral interpretation of sinusoidal wave using Fractional Fourier transform based FIR window Function. International review on computers and software (I.RE.CO.S), Vol.4, No.6, November 2009.

[6] P.V.Muralidhar, D.Nataraj,V.Lokesh Raj \& S.K.Nayak “” Implementation of high-pass filter using Fractional Fourier Kaiser window",IEEE-explore Aug.2010,ISBN-978-1-4244-68928,vol.2,pp.651-655.

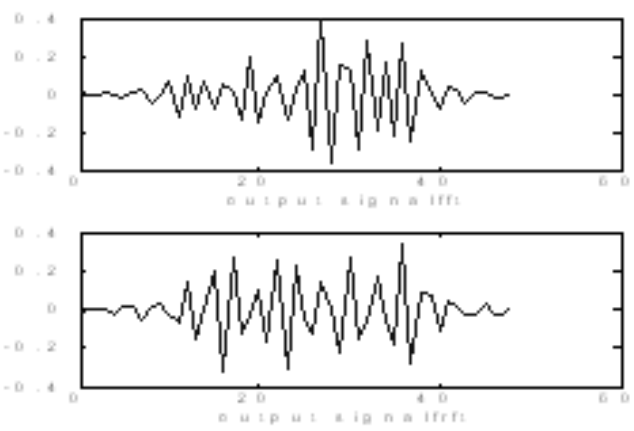

SNRLOWFRFT $=2.6319$

Fig. 5.11.b

$\mathrm{a}=1$
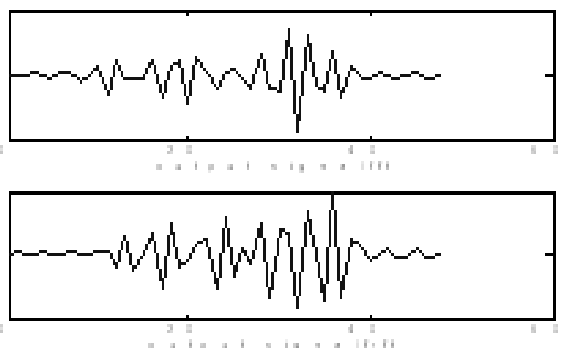

SNRLOWFRFT $=2.4865$

Fig. 5.12.b

[7] Namias.V-"The FrFT and Time Frequency representation"J.Inst.Math.Applications, Vol:25,pp.241-265,1980. 